\title{
An electrically tunable filter based on a long-period fiber grating with a thin liquid crystal layer
}

\author{
Aleksandra Czapla, ${ }^{* 1,3}$ Tomasz R. Woliński, ${ }^{1}$ Roman Dąbrowski, ${ }^{2}$ Edward Nowinowski-Kruszelnicki ${ }^{2}$, \\ and Wojtek J. Bock ${ }^{3}$ \\ ${ }^{I}$ Faculty of Physics, Warsaw Univ. of Technology, ul. Koszykowa 75, 00-662 Warszawa, Poland \\ ${ }^{2}$ Military Univ. of Technology, ul. Gen. Sylwestra Kaliskiego 2, 00-908 Warszawa, Poland \\ ${ }^{3}$ Centre de recherché en photonique, Univ. du Québec en Outaouais, Rue Saint-Jean-Bosco 101, J8X 3X7, \\ Gatinau, Canada
}

Received September 22, 2010; accepted September 28, 2010; published September 30, 2010

\begin{abstract}
In this paper we report experimental results concerning electrical tuning of a long period grating (LPFG) using as its external layer a liquid crystal (LC). The studied LPFG was fabricated by using UV irradiation in a boron co-doped fiber (PS1250/1500, manufactured by Fibercore). As an LC we used the 1550 nematic LC mixture. Electrical tuning of the LPFG transmission spectrum is achieved by varying the boundary condition of the LC layer. As a result, a blue shift of the attenuation band up to $6.2 \mathrm{~nm}$ was obtained.
\end{abstract}

A long period grating (LPFG) [1-2] is a grating with a pitch of several hundred micrometers formed in an optical fiber in order to achieve light coupling between the fundamental core mode and the forward propagating cladding mode. As a result, the transmission spectrum of the LPFG is characterized by a series of attenuation bands. The transmission characteristics of the LPFG formed in a single-mode fiber can be analyzed by the coupled-mode theory [1]. The spectral position and intensity of these attenuation bands depend on temperature, strain, bend or surrounding refractive index (SRI) changes. For these reasons filters based on LPFGs have generated a significant interest in the sensing applications. Compared to other optical devices, LPFGs have a number of unique advantages such as low-level back reflection, low insertion losses and compact construction. According to the SRI sensitivity of an LPFG, liquid crystals (LCs) can be used as a surrounding material of an LPFG since it can offer a form of dynamic spectral control [3-5]. The properties of LCs provide tunable action of their refractive indices by temperature, magnetic or external electric filed changes. There are three key points for a good design in order to combine an LPFG and an LC into one component: selection of an LC characterized by desirable refractive indices, inherent SRI sensitivity of an LPFG, and LC layer thickness. So far, most of the studies have focused on the analysis of SRI sensitivity when the LPFG cladding is surrounded by a
LC medium of infinite thickness. The first evidence of LPFG tuning by using an LC was demonstrated by Duhem et al. [3]. They proposed electrical modulation of an LC around a photo-induced LPFG. Furthermore, a cascade structure of LPFGs with an LC as a surrounding medium was proposed by H-R Kim et al. [4], for arbitrary loss filters that compensate non-uniform optical gain in an erbium-doped fiber. However, the tuning range of the transmission spectrum of LPFGs with LCs presented in [3] and in [4] was limited mainly to the controlling of the intensity of attenuation bands by an electric field across the LC surrounding. This is due to the fact that the majority of LCs have refractive indices that are higher than the refractive index of silica glass. Therefore, the LPFG sensitive to SRI is limited to the range where SRI is higher than the refractive index of the fiber cladding. In this case, the core modes couple to leaky modes and sufficient tuning of resonant wavelengths of an LPFG versus the SRI is not so straightforward [6]. In addition, modification of the depth of attenuation bands is generally expected. Strong improvement in the LPFG SRI sensitivity can be obtained by reducing the thickness of the high refractive index (HRI) medium [7, 8]. Due to the refractive-reflective regime at the cladding-LC layer interface, the cladding modes in an LPFG surrounded by an HRI layer are bound within the structure comprising the core, the cladding, and the layer. The idea of an LPFG coated with a thin LC layer (order of $1 \mu \mathrm{m}$ ) was brought forward by Luo et al. [9]. Experimental results show that by inducing a change of temperature in the LC refractive index, a shift greater than $80 \mathrm{~nm}$ in the attenuation bands of the transmission spectrum LC-coated LPFG was achieved.

In this paper we demonstrate a high-efficiency electrically tunable filter based on the LPFG surrounded by a layer of the LC mixture.

*E-mail: czapla@if.pw.edu.pl 
For the needs of the present work, the LPFG was fabricated by UV irradiation in a boron co-doped photosensitive fiber (PS1250/1500, manufactured by Fibercore [10]). The PS1250/1500 fiber gives a possibility of writing the grating directly, without any hydrogenation procedure. A segment of PS1250/1500 fiber, with polymer coating removed, was spliced between two sections of a standard communication fiber (SMF28, manufactured by Corning) and placed in contact with the amplitude mask having a periodicity of $227 \mu \mathrm{m}$. The source of the UV light was provided by an Eximer laser (PulseMaster GSI Lumonics) emitting at the $248 \mathrm{~nm}$ wavelength. Consequently, the fiber was irradiated by UV light and the exposure was repeated until the index modulation reached a sufficient level to provide the desired attenuation depth in the LPFG transmission spectrum. The length of the LPFG was $4 \mathrm{~cm}$.

As an LC material we used a low birefringence nematic LC mixture 1550 synthesized at the Military University of Technology in Warsaw, with the ordinary, $n_{o}$ and extraordinary, $n_{e}$ refractive indices 1.46 and 1.52 , correspondingly (measured at $587 \mathrm{~nm}$ and at $23^{\circ} \mathrm{C}$ ). In order to form a LC layer on the surface of the LPFG, first, the LPFG was rubbed several times along the fiber axis so that the LC molecules tend to align along the fiber axis. In reality, there exists certain non-zero tilt angle of LC molecules with respect to the fiber axis, which depends on the geometry of the LC layer as well as the fiber itself. However, the overall effect can be negligible so that the effective refractive index of the LC layer can be approximated by $n_{o}$ of the 1550 LC. Next, the LPFG was coated with the LC layer. Surface tension of the LC makes it easy to uniformly coat the LPFG making the LC behave as if it were a skin layer. The presence of the LC layer on the surface of the LPFG was observed when the sample was placed between the parallel polarizers. Since birefringence of the $1550 \mathrm{LC}$ is known, the LC layer can be estimated to an order of $1 \mu \mathrm{m}$ - according to the Michel-Levy interference chart [11]. The transmission spectrum of the sample was investigated with the input light projected from a halogen lamp/super continuum source and the output signal was analyzed by an Optical Spectrum Analyzer.

In Figure 1, the measured spectra of the LPFG in air and with a layer of the $1550 \mathrm{LC}$ mixture at $25^{\circ} \mathrm{C}$ are presented. In the transmission spectrum of the LPFG in air, three main attenuation bands can be seen, designated by Band 1, Band 2 and Band 3. When a bare LPFG is coated with the $\overline{1} 550$ LC mixture, a flattening of the Band_3 is observed. In addition after coating the LPFG with the LC layer, the Band_1 and the Band_2 turned into one band with resonant wavelength at $1229 \mathrm{~nm}$.

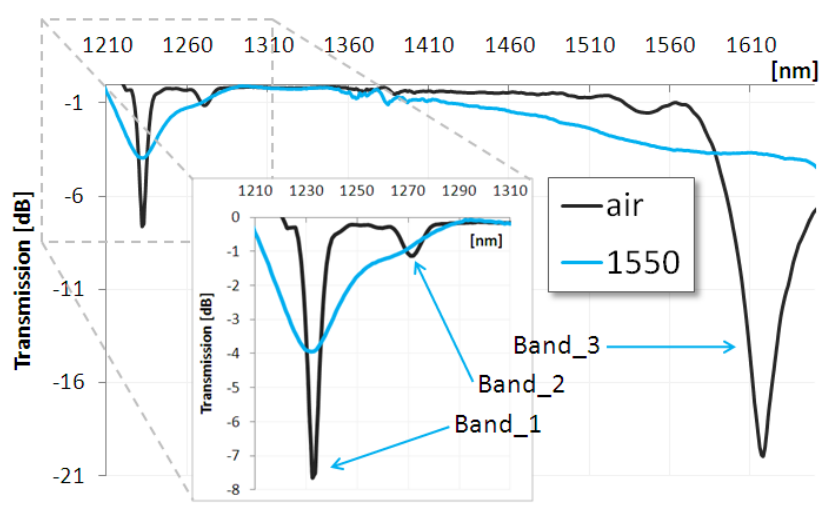

Fig. 1. Transmission spectra of the LPFG in air and with 1550 layer.

The next step consisted in placing an LPFG with the 1550 layer between two electrodes. The space between electrodes was $140 \mu \mathrm{m}$. Electrical control was conducted in the $0 \mathrm{~V} / \mu \mathrm{m}$ to $4 \mathrm{~V} / \mu \mathrm{m}$ range. The key fact is that optical properties of an LPFG depend strongly on the refractive index of the LC layer and consequently on the orientation of the 1550 LC mixture molecules. Without an electric field, the LC molecules are aligned along the fiber axis and an effective refractive index of the LC layer corresponds to $n_{o}$ of $1550 \mathrm{LC}$. Under the influence of an external electric field, molecular reorientation occurs. Then, at voltages higher than the threshold value (according to the Fredericks transition rule) molecules tend to orient perpendicularly to the fiber axis.

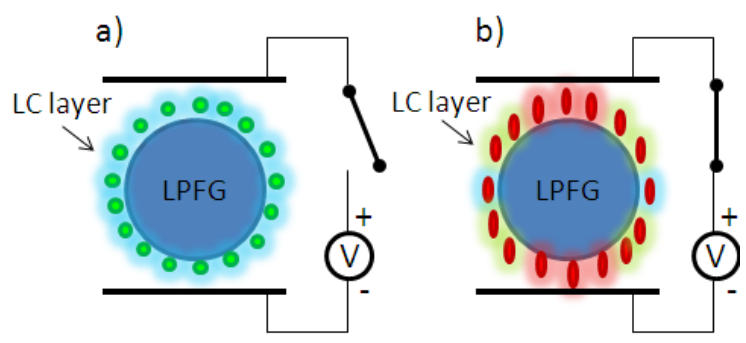

Fig. 2. The cross section of two geometries of the alignment of LC molecules around the fiber: a) planar b) unidirectional.

If the alignment of the LC molecules is changed from planar to unidirectional (like is shown in Fig. 2), the effective index of the LC layer is changed and the effective index of the cladding region of the LPFG is changed as well. As a consequence, the tunable action of the attenuation band in the LPFG transmission spectrum is achieved as is illustrated in Fig. 3. An electrical field induces wavelength shifts and modification of the depth of an attenuation band for the LPFG with the 1550 LC layers are plotted in Fig. 4. It seems that under an external electric field a blue shift of an attenuation band occurs, up to $6 \mathrm{~nm}$. An electrically induced modification of the transmission spectrum of LPFG with 1550 LC layer starts 
occurring around $0.89 \mu \mathrm{m} / \mathrm{V}$. In addition, an increase in the depth of an attenuation band at a sufficiently high value of an external electric field (above $1.82 \mu \mathrm{m} / \mathrm{V}$ ) was recorded.

$\begin{array}{llllllll}1205 & 1215 & 1225 & 1235 & 1245 & 1255 & 1265 & 1275\end{array}$

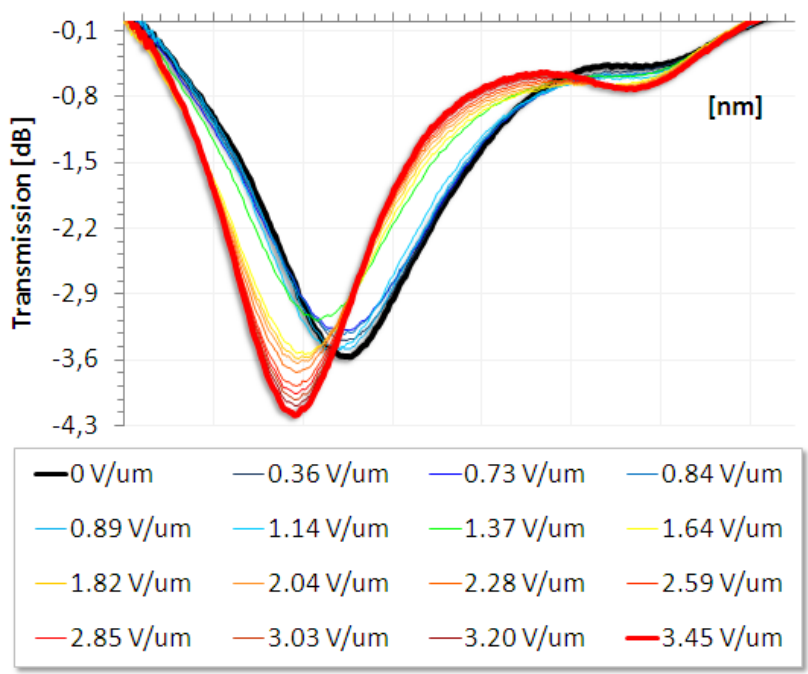

Fig. 3. Transmission spectrum of an LPFG with the 1550 layer versus an external electric field.

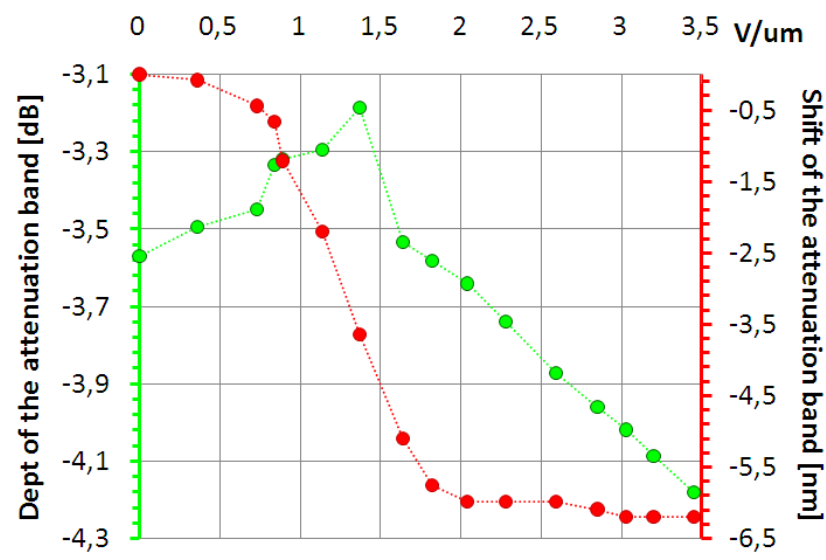

Fig. 4. The electrical field induces wavelength shifts and modification of the depth of an attenuation band for an LPFG with the 1550 LC layer.

To conclude, the experimental results that were obtained have demonstrated that the presence of the LC as an active material enhances functionality of the LPFGs and could be applied toward further development of practical tunable optical filters with an electrical sensitivity. In previous publications [3-5] related to LPFG surrounded by $\mathrm{LC}$, the electrically induced tuning range of it transmission spectrum was mainly limited to the controlling of the intensity of attenuation bands by an electric field across the LC surrounding. Our experiments have shown that by reducing the thickness of the high refractive index LC medium to order of $\sim 1 \mu \mathrm{m}$, electrically induced changes in the effective refractive index of the thin LC layer also led to a considerable shift of the attenuation band in the transmission spectrum of the LPFG. In addition it has to be reminded that, LCs are anisotropic materials and have uniaxial birefringent properties. Therefore, when the electrical field is applied, light waves polarized perpendicularly to the fiber axis experience the $n_{e}$ of 1550 . On the other hand, light waves polarized parallel to the fiber axis will experience the $n_{o}$ of 1550. As a result, the LPFG for which the LC layer is in such configuration of electrodes should also exhibit the sensitivity to the polarization state of the light. This note indicates that the polarization properties of LPFG with LC layer should also be studied in further research.

This work was supported by the Natural Science and Engineering Research Council of Canada, by the Canada Research Chairs Program. The authors are also grateful to the Foundation for Polish Sciences (FNP) Professor's Subsidy „MISTRZ”, to the European Union in the framework of the European Social Fund through the Warsaw University of Technology Development Program, and to the Fonds québécois de la recherche sur la nature et les technologies (FQRNT).

\section{References}

[1] T. Erdogan, J. Opt. Soc. Am. A 14, 1760-1773 (1997).

[2] S.W. James and R.P Tatam, Meas. Science and Technol. 14(5), R49R61 (2003).

[3] O. Duhem, J.F. Henniont, M. Warenghem, M. Douay, L. Rivoallan, Proc. 6th IEE Conf. on Telecommunications 451, pp. 195 (1998).

[4] H-R. Kim, Y. Kim, Y. Jeong, S. Baek, Y. Wook Lee, and S-D. Lee, Mol. Cryst. Liq. Cryst, 413, 399-406 (2004).

[5] A. Czapla, W.J. Bock, T. Woliński, and P. Mikulic, Phot. Lett. of Poland 1(2), 100-102 (2009),

http://photonics.pl/PLP/index.php/letters/article/view/1-34/36.

[6] R. Hou, Z. Ghassemlooy, A. Hassan, C. Lu, and K. P. Dowker, Meas. Sci. Technol. 12, 1709-1713 (2001).

[7] I. Del Villar I. R. Matias, and F. J. Arregui, IEEE Photon. Technol. Lett. 17, 1893-1895 (2005).

[8] A. Cusano, A. Iadicicco, P. Pilla, L. Contessa, S. Campopiano, and A. Cutolo, Opt. Exp. 14, 19-34 (2006), http://www.opticsinfobase.org/oe/abstract.cfm?URI=OPEX-14-119 .

[9] H. Luo, X. Li, S. Li, and J. Chen, Appl. Opt. 48, F95-F100 (2009).

[10] Fibercore product factnote for PS1200/1500 fiber http://www.fibercore.com/Portals/0/PropertyAgent/576/Files/266/P S\%20Fiber.pdf

[11] J. G. Delly, on-line journal Modern Microscopy (2003), http://www.modernmicroscopy.com/main.asp?article=15\&page=1 